Décadrages Décadrages

cinéma, à travers champs Cinéma, à travers champs

6 | 2005

Train et cinéma

\title{
Entretien avec Nicolas Humbert et Werner Penzel
}

\section{Marthe Porret et Laura Legast}

\section{(2) OpenEdition}

Journals

Édition électronique

URL : https://journals.openedition.org/decadrages/506

DOI : $10.4000 /$ decadrages.506

ISSN : 2297-5977

\section{Éditeur}

Association Décadrages

\section{Édition imprimée}

Date de publication : 10 octobre 2005

Pagination : 112-121

ISBN : 978-2-9700582-2-9

ISSN : 2235-7823

Référence électronique

Marthe Porret et Laura Legast, «Entretien avec Nicolas Humbert et Werner Penzel », Décadrages [En ligne], 6 | 2005, mis en ligne le 20 janvier 2014, consulté le 27 mars 2022. URL : http://

journals.openedition.org/decadrages/506 ; DOI : https://doi.org/10.4000/decadrages.506

(B) Décadrages 


\title{
Entretien avec Nicolas Humbert et Werner Penzel
}

\author{
par Marthe Porret et Laura Legast
}

\section{Réaliséle 23 avril 2005 à Nyon, dans le cadre du festival Visions du Réel}

Werner Penzel, né en Allemagne en 1950, étudie le cinéma à la Hochschule für Film und Fernsehen de Munich, de 1971 à 1975. Né en 1958, le Suisse Nicolas Humbert le rencontre quelques années plus tard, y étant lui-même étudiant de 1982 à 1987. En 1987, tous deux fondent la maison de production Ciné Nomad et signent deux ans plus tard leur premier film commun: Lani und die Seinen. En 1990, ils réalisent Step Across the Border qui, projeté dans de nombreux festivals internationaux, remporte plusieurs prix. Suivent deux années de tournage auprès des Touaregs du Sud qui donneront Middle of the Moment, en 1995. Ils signent en 1997 Nulle Sonne. No Point, un documentaire consacré au Chicago Art Ensemble. Régulièrement en visite chez le poète Robert Lax entre 1993 et 1999, ils lui consacrent une installation sur trois écrans: Three Windowes, dont la version film s'intitule Why Should I Buy a Bed when All I Want Is to Sleep (2001).

En voyant votre dernier film, Brother Yusef (2005), on a un peu eu l'impression d'un film-synthèse. On y retrouve à la fois votre intérêt pour la musique, que vous aviez si bien captée dans Step Across the Border, et une grande ressemblance formelle avec Three Windows...

Nicolas Humbert: Comme je l'ai dit après la projection, quand on choisit un thème, il y a déjà une certaine logique et une certaine esthétique dans ce choix. Nous ne nous sommes pas dit: maintenant nous voulons continuer dans ce type de film, cette esthétique, ce genre de thème. Mais bien sûr, il y a quelque chose de général qui nous préoccupe, certainement, oui. Si l'on regarde rétrospectivement son œuvre, des choses se cristallisent, des traces restent. On peut tirer des liens entre Three Windows et Brother Yusef. Non seulement parce qu'il s'agit de deux hommes âgés, mais surtout grâce à cet espace intérieur qu'on essaie de rendre visible par des moyens cinématographiques. C'est notre revendication, je crois, d'être intéressés par quelque chose de profond, d'intérieur. Au début, il y a toujours une sorte de résistance, c'est cette sorte de tension qui nous intéresse et qui forme le film. Mais rien n'était sûr au début.

Il y a une très grande différence formelle entre un film comme Step Across the Border et Brother Yusef, tous deux des portraits de musicien. Cela découle-t-il de la personnalité de Yusef Lateef et de celle de Fred Frith? 
Werner Penzel: Step Across the Border n'est pas le portrait d'un musicien! L'idée n'a jamais été de faire un portrait de Fred Frith. Par ailleurs, je pense que si on avait approché Fred en lui disant que nous voulions faire un portrait de lui, il nous aurait envoyé ballader! Ce qui s'est passé, c'est qu'avec Fred, nous avions une façon d'appréhender les images et les sons qui ressemble à son approche du son. Pour Frith, on peut créer un univers à partir de toutes sortes de types de sons, comme on peut dire que tout son est musique. C'est comme ça qu'il compose. De la sorte, nous avons travaillé avec Fred à la façon d'un trio: il donnait quelque chose, nous donnions quelque chose, et nous avons concocté une soupe de cette marmite!

Avec Yusef, il faut savoir comment cela a commencé. C'est en fait un ami saxophoniste qui connaissait très bien notre travail qui est venu nous trouver un jour en nous parlant de Yusef Lateef, que nous ne connaissions alors qu'à travers sa musique. Autrement, nous n'avions aucune idée de qui était Yusef Lateef! Il nous a parlé de l'urgence de faire un film sur ce musicien de 84 ans, une personne tellement incroyable, un homme magnifique. "Il faut absolument faire quelque chose sur lui! Il ne sera bientôt plus là.» Cet ami a tellement insisté qu'un jour nous avons accepté. Nous avons pris l'avion, pour lui rendre visite, chez lui, mais sans emporter de caméra, ni de micro. Nous avons pris le thé avec lui dans son salon. Et nous avons été très impressionnés: c'est un homme merveilleux, très humble.

Votre film est précisément un film sur l'bumilité. C'est frappant: un documentaire sur l'bumilité.

N. H., W. P.: Nous avons tout simplement suivi cette proposition. Parfois, certaines choses arrivent et vous vous demandez pourquoi je devrais faire ça, et... Si notre ami n'avait pas autant insisté, nous n'aurions pas suivi cette piste. Et tout a été payé de notre poche, nous n'avions aucune production, rien. C’était simplement «OK, prenons l'avion, allons rendre visite à frère Yusef et regardons ce qui se passe entre nous à ce moment-là!»

\section{Dans quelles conditions avez-vous produit ce film?}

W. P.: Il était clair que ce serait un présent magnifique que de pouvoir faire quelque chose avec lui, c'est tout ce que nous savions, vraiment tout. Et puis nous avons eu des discussions avec la télévision, et

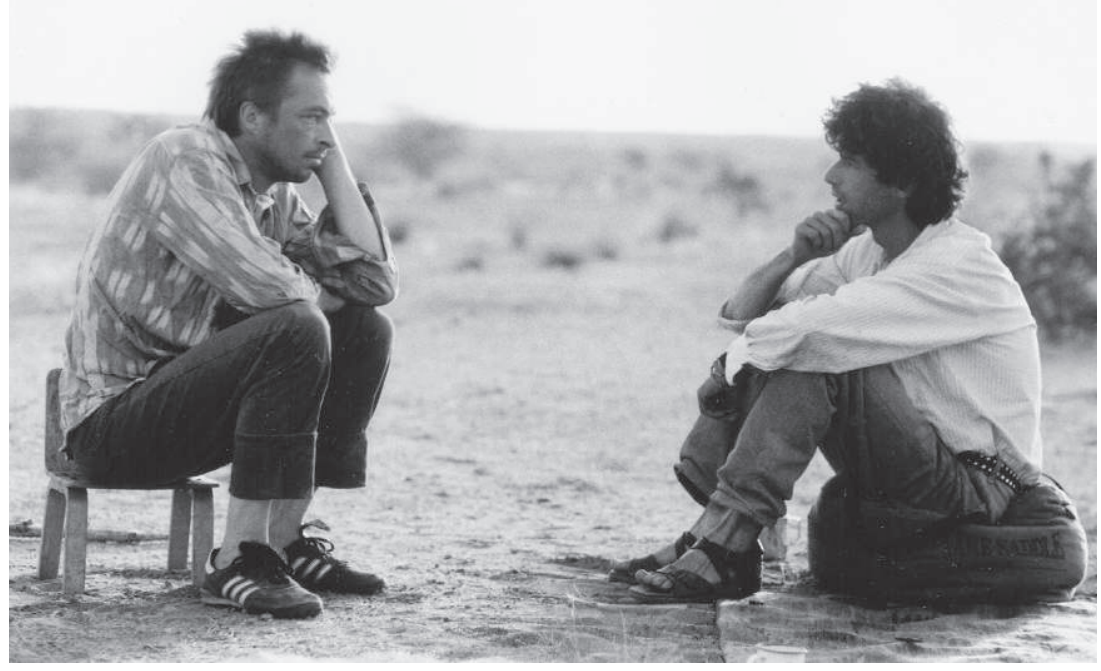



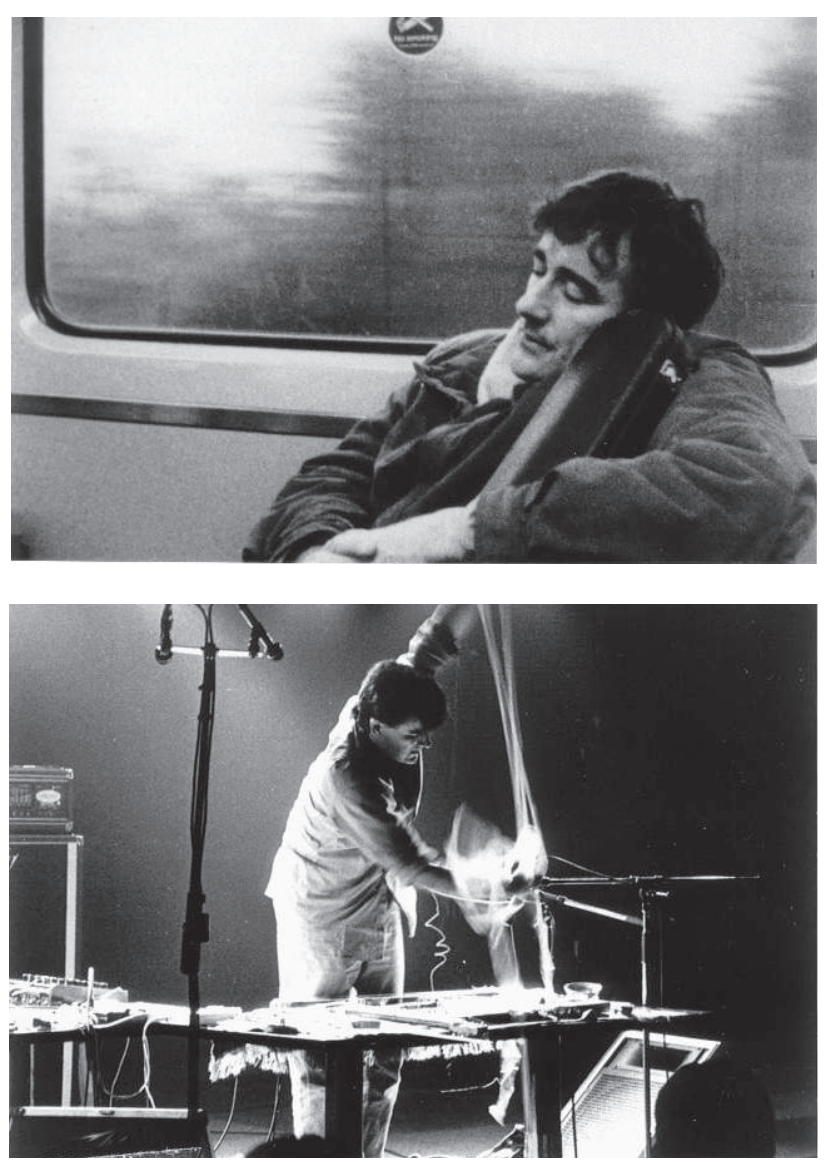

on nous disait sans cesse: oui, peut-être... Toujours ce même genre de réaction attendue. Tout le monde sait que vous venez avec tout votre cœur, vous le posez sur la table et tout le monde le regarde comme si c'était un morceau de saucisse (rires), ouais, c'est comme ça! Et puis, il est arrivé un moment où il est devenu clair que si nous ne prenions pas nous-mêmes une petite caméra et un équipement son, le projet allait s'évanouir lentement. Alors nous nous sommes rendus là-bas et, comme avec Robert Lax, nous avons rencontrés Yusef Lateef tous les jours pendant deux, trois heures, parfois le matin, parfois l'après-midi, et puis il a senti que c'était une sorte de défi. Et tous les jours il était préparé; quand nous arrivions à la maison nous pouvions l'entendre depuis très loin en train de jouer de son saxophone, en train de se chauffer! Et alors il jouait, jouait, jouait et jouait... Nous lui avons dit qu'il ne devait pas, mais il voulait, il voulait vraiment...

A-t-il tout de suite accepté? Avez-vous dî le convaincre? N. H.: Nous avons apporté un dossier de Three Windows et nous lui avions envoyé Step Across the Border et Middle of the Moment, pour qu'il se fasse une idée de notre approche. Parce qu'il était très timide, très timide. Comme il a choisi une vie très cachée, dans 
la forêt, très loin de tout, il n'est pas intéressé par le fait d'être filmé. Donc, il n'avait aucun intérêt à être filmé. Mais peut-être qu'il a senti... Créer quelque chose dans un délai aussi bref est très mystérieux: on se rencontre, on doit faire comprendre qu'on ne travaille pas pour la TV, ou pour de l'argent, mais qu'on s'intéresse simplement à un être humain. En fait, c'est toujours la base de notre travail, je crois. Quelque chose se passe ou non. Quand nous sommes rentrés, nous avions le sentiment que ça avait été beau d'être tout simplement là avec lui. Nous n'avions aucune idée de ce que serait le film, mais seulement un sentiment général de vouloir continuer sur cette idée.

\section{Ses improvisations musicales étaient-elles complètement libres?}

N. H.: Nous discutions tous les jours avec lui de ce que nous allions faire le jour suivant. Il disait: OK, j'ai le temps entre deux heures et six heures. Vous pouvez venir à deux heures. Nous décidions avec lui de commencer par du saxophone par exemple. Il se préparait à avoir une session l'après-midi avec son saxophone. Et quand nous arrivions nous entendions déjà le saxophone par les fenêtres! Puis il entrait en transe, une transe qu'on pouvait sentir. Il soufflait dans son saxophone pendant quatre heures, pratiquement sans s'arrêter. Et nous, nous étions dans la pièce, sans l'interrompre, à chercher le bon emplacement de la caméra et de l'équipement sonore, doucement. Nous le laissions simplement dans sa transe. Il faisait ce qu'il avait à faire. Il était dans son propre monde. C'est peut-être aussi ça la particularité de ce film, que de pouvoir sentir cette transe, de sentir qu'il revient de cet espace intérieur. Et nous avons préparé ça tous les jours. Le jour suivant nous lui proposions de faire quelque chose autour de la poésie, parce qu'il est aussi poète. Alors il se préparait. Ou parce que nous connaissions ses recueils, nous lui suggérions un poème qui nous intéressait, et il préparait cet après-midi ou cette matinée de lecture. Chaque jour avait un certain thème. $\mathrm{Ou}$ alors, comme nous savions qu'il joue aussi du piano et qu'il chante - nous avions assisté à un concert où il jouait du piano - nous lui avons demandé de le faire. Il a répondu que son piano n'était pas accordé, qu'on ne pouvait pas l'utiliser! Nous étions désolés car sa voix et sa façon de chanter sont très impressionnantes. Mais le jour d'après, c'était possible! Il avait trouvé quelque chose. Il était ouvert, vous savez. Il nous donnait une feuille de papier avec tous les titres. Il avait retranscrit durant la nuit tout ce qu'il avait composé... C'était sérieux, vous savez. Je crois qu'il comprenait la situation, la possibilité qu'il avait de donner encore une fois quelque chose de son univers à un public. Voilà comment s'est structurée notre rencontre avec lui. Chaque jour un certain thème, comme une jam session.

\section{Combien de temps êtes-vous restés là-bas?}

Deux semaines seulement. Ce qui est long et court à la fois.

\section{Deux semaines en filmant tous les jours?}

Plus ou moins, oui. Un jour, il nous a aussi invités à venir avec lui à la mosquée. Car, tous les vendredi il va à Boston, qui se trouve à trois heures de voiture de là. La religion, la spiritualité sont la base de sa vie. Il nous a donc invités à l'accompagner à la mosquée. Nous y avons tourné, mais à la fin il était clair que ce ne serait que chez lui, rien à l'extérieur. Nous avons aussi pensé organiser une rencontre avec ses vieux amis musiciens, comme Archie Shepp et Sonny Rollins qui vivent tout près, ou le suivre en concert - il joue encore de temps en temps. Mais après ces deux semaines, nous avions le sentiment clair que cette forme concentrée constituerait le film. Nos films sont un travail en cours, c'est toujours un work in progress.

Etait-il clair dès le début que vous ne vouliez pas donner d'informations biographiques au spectateur?

W. P.: Oui. Je pense que cela aurait détourné l'attention de ce qui constitue selon moi l'essentiel de ce film. Peut-être ce film a-t-il une qualité : c'est que vous 
BROTHER YUSEF (2005)

plongez vraiment dans l'intensité d'une personne, dans son ressenti. Dès le moment où vous commencez à dire : il est né là, a joué avec tel et tel, a grandi là, puis a déménagé à New York, est devenu professeur, etc., vous vous focalisez dans votre esprit sur ces informations et vous commencez à voir à travers ces lunettes-là, à travers ces informations qui sont toujours fragmentaires. Et le simple regard qu'un enfant peut avoir sur le monde, simplement voir et ne pas juger, ne pas mettre dans des cadres, juste voir ce qu'il y a, cette sorte de possibilité de regarder quelqu'un ou le monde, vous le détruisez en commençant par donner des informations. Ensuite, vous ne pouvez plus regarder avec ce regard innocent ou plus direct.

Un regard innocent, est-ce votre définition du documentaire, de la façon dont vous faites des documentaires en général?

N. H.: C'était très beau, ce que vous avez dit au début. Vous avez dit: ce n'est peut-être pas un portrait, mais un film qui traite d'humilité. C'est ça. C'est ce qui touche, je pense, le cœur de notre travail. Il s'agit toujours d'êtres humains que l'on suit, et le but n'est pas d'en faire le portrait, mais d'exprimer des choses qui sont plus générales.

Vous offrez ainsi une grande liberté d'interprétation au spectateur.

N.H. : Nous essayons toujours de laisser aux spectateurs

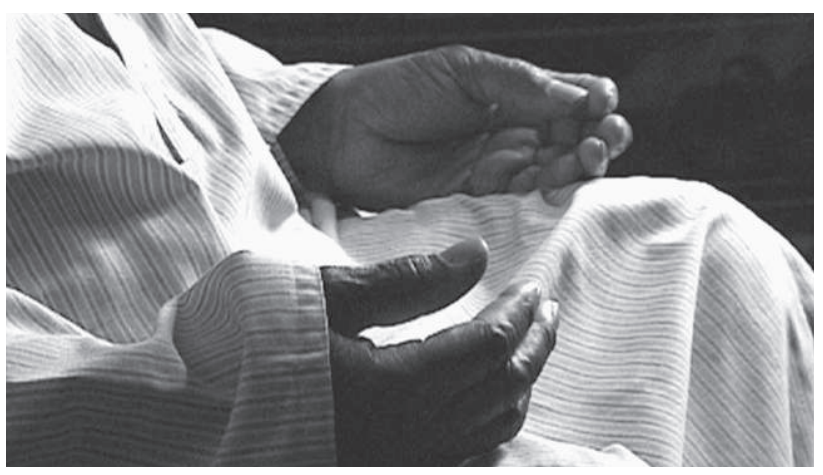

le plus possible de liberté. Comme Werner l'a dit, la difficulté réside dans le fait de donner des informations. Avec des informations factuelles, tu es toujours dans un cadre de télévision, ce qui se ressent aussi esthétiquement. Si tu commences avec des explications, tu transformes la forme d'un film. C'est certainement ce qui constitue le caractère général de nos films. Il y a parfois des gens qui veulent en savoir plus. Pour eux, les points de critique portent précisément sur le fait que rien n'est expliqué. Nous avons toujours ce genre de discussion après les films. Avec Middle of the Moment spécialement, par exemple, car le spectateur ne sait pas où nous sommes par exemple... Mais c'est là notre manière de faire.

Vous n'avez presque pas filmé les détails de la vie quotidienne...

W. P.: Nous les trouvons dans le film, bien sûr. Ce n'est pas que nous ne sommes pas intéressés par les choses quotidiennes. Pas du tout, pas du tout! Plusieurs de nos films traitent énormément de cela! Dans ce cas précis, il ne s'agissait pas de capter des détails. Il y en a: quand il boit son café et mange un beignet, par exemple. Mais ce n'était définitivement pas le centre d'intérêt du film. Le premier jour de tournage, il a voulu savoir à quel genre de film notre travail commun aboutirait. Il voulait connaître notre avis sur la question. Je suppose qu'il y a eu, comme d'habitude, d'abord un silence, et puis nous avons dit: peut-être 
pouvons-nous faire quelque chose ensemble comme prier pour la paix. C'était l'époque de la guerre en Irak, et d'une certaine manière nous étions aux EtatsUnis avec un noir, musulman, et ces événements occupaient nos esprits. C'était donc très naturel de dire ça, sans se forcer: nous sommes ici dans cette pièce et peut-être pouvons-nous faire quelque chose comme prier ensemble pour la paix. Il a très bien compris cela. Je pense qu'il était très content. Nous nous sommes rencontrés dans cette pièce, nous avons concentré nos prières pour la paix, sans le dire explicitement mais par la façon dont nous avons procédé, tout naturellement, paisiblement.

\section{Le film donne l'impression que Yusef Lateef est un} bomme très solitaire, une sorte d'ermite. Est-il comme ça dans la vie?

W. P.: C'était en fait sa décision. Il vit avec une femme
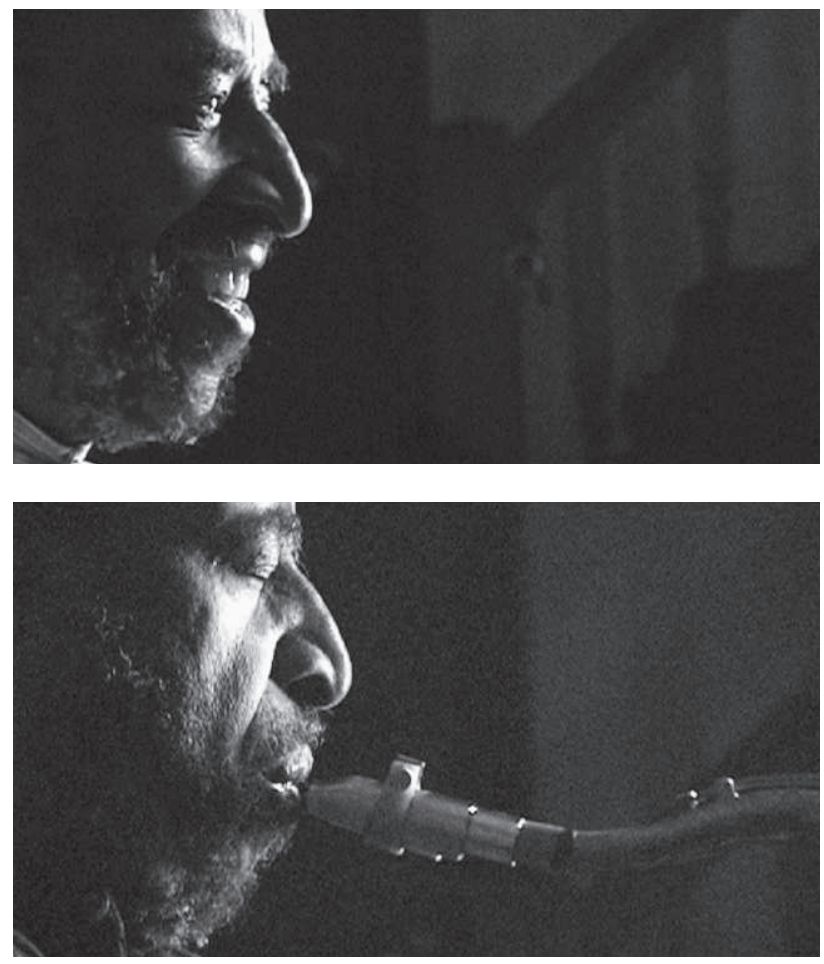

extraordinaire, très forte, et il a un fils de sa seconde femme qui vit au-dessus et qui étudie à l'université. Mais avant que nous commencions, il nous a dit: je ne veux pas que ma femme, ni mon fils soient filmés. C'est une décision claire que nous devions respecter tout simplement.

N. H.: Le fait qu'il n'y a pas de vie domestique dans ce film, cela donne finalement lieu à une certaine logique, à une certaine qualité de concentration. Mais ce n'était pas délibéré de notre part, cela s'est décidé à travers lui. C'est bizarre, parfois cela vient de l'extérieur, et à la fin, vous vous rendez compte que c'était tout à fait juste. Mais ce n'était pas notre décision.

\section{Le film est divisé en dix chapitres. Comment sont-ils nés?} Les aviez-vous en tête avant le tournage?

N. H. : Le montage n'était pas facile. Le matériel offrait peu de choix. La plupart des plans sont des gros plans 

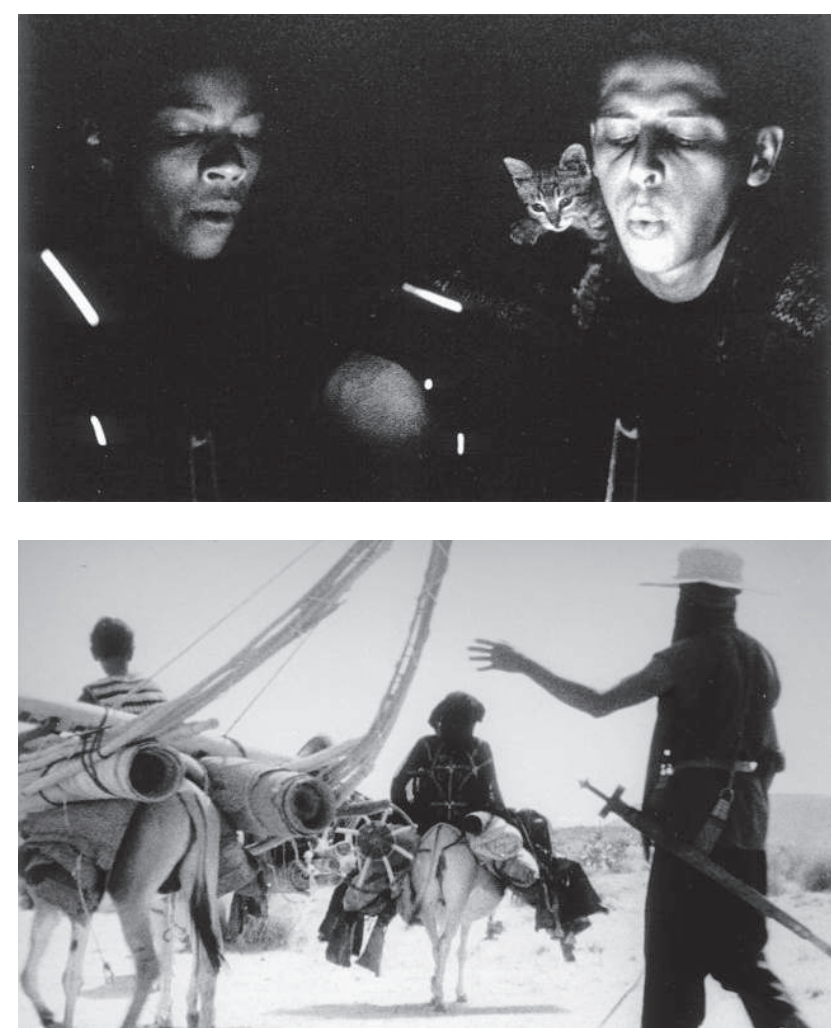

du visage de Yusef Lateef. Alors comment peut-on structurer un film qui travaille principalement avec ce type de plans? Il fallait trouver une solution, essayer d'utiliser des mots pour trouver une structure; le noir est un moyen de ponctuation, aussi. En plus des chapitres, il y a aussi le noir uniquement, sans chapitre. C'est quelque chose qui se décide au coup par coup, qui n'est pas fixé dès le début.

Le chapitre "Remembrance», par exemple, vient du fait que Lateef fait des liens: il parle de son amitié avec John Coltrane, puis on l'entend jouer. Remembrance est peut-être un souvenir musical de ce qu'il nous a dit de son amitié avec John Coltrane. Il essaie constamment de donner quelques petits indices, mais sans jamais décrire ce qui va venir. Il s'agit de créer des ponts. En fait, ce ne sont pas des chapitres, mais

des ponts. Des ponts entre des thèmes et dans une manière libre. Il ne s'agit pas de décrire au moyen de titres, mais de trouver des liens entre les phases, les différents épisodes.

Comment partagez-vous le travail au moment du tournage?

W. P.: Quand il faut dire qui fait quoi durant le tournage, nous répondons que Nicolas s'occupe de l'équipement sonore et que je suis en charge de l'image. Mais c'est trompeur. Dire: il est le preneur de son, je suis le caméraman, fausse la compréhension de la manière globale dont nous travaillons. Nous sommes deux personnes très différentes, notamment du point de vue de nos énergies. Mais à nous deux, nous créons un espace qui n'est ni le sien ni le mien. Je ne dis pas 
que c'est notre espace, c'est seulement un espace dans lequel tout arrive. Quand nous travaillons ensemble, nous créons un certain type d'attention, de concentration, une émotion si vous voulez, qui n'est ni la sienne ni la mienne. C'est un cadeau qui nous est donné de pouvoir faire ça. Vous pouvez le vouloir, mais il y a tant de choses complètement irrationnelles qui se passent alors, qu'il ne sert à rien de dire: je veux que ça arrive! Ça arrive, c'est tout.

N. H.: Nous avons un sentiment commun quand le moment est là. Nous ne discutons jamais pour décider quand il faut commencer à tourner. C'est un sentiment qui se cristallise au même moment chez chacun de nous. C'est un cadeau qui nous est donné de pouvoir partager ça.

\section{Au fil des films, votre manière de travailler ensemble a-t-elle changé? Une plus grande complicité s'est-elle développée?}

W. P.: Cela a été comme ça dès la première fois, dès que nous sommes allés d'Allemagne au Japon, pour commencer à tourner Step Across the Border, et ça a toujours été comme ça. Comme je l'ai déjà dit, l'intensité et la qualité de cet espace - bien qu'il ne soit jamais le même - n'ont jamais changé. C'est simplement comme ça.

N. H.: Je pense que c'est quelque chose que les personnes que nous approchons ressentent. Quelqu'un comme Yusef Lateef, ou Fred, sentent qu'il n'y a pas deux personnes qui doivent discuter de ce qu'ils vont faire, mais qu'ils forment simplement un espace où tout le monde va pouvoir se rencontrer. C'est peut-être cette intimité aux choses qui existe dans tous nos films.

W. P.: Nous sommes très différents, mais nous ne sommes pas des concurrents.

\section{Qu'en est-il de votre travail individuel?}

N. H.: Au cours de ces vingt dernières années, les œuvres intermédiaires ont été réalisées individuellement, mais les films plus importants ont toujours été faits ensemble. Il y a bien sûr des domaines qui concernent plus l'un ou l'autre, mais durant le tournage nous nous retrouvons complètement.

W. P.: Et nous ne sommes pas du genre à faire quelque chose si l'un de nous a des doutes à ce sujet. Si nous pensons tous deux qu'un nouveau projet est bon, nous le réalisons. Nous n'avons pas non plus besoin de longues discussions à ce sujet.

\section{Pour Brother Yusef, d'autres personnes que vous fai-} saient-elles partie de l'équipe?

N. H.: Non, nous n'étions que les deux. Sur la plupart de nos tournages, nous ne sommes que les deux. Pour Step Across the Border, nous étions trois, il y avait quelqu'un qui était assistant général. Sur Middle of the Moment, nous étions quatre, parce que c'était un tournage un peu plus organisé. Mais avec Robert Lax par exemple, et aussi avec Yusef, nous n'étions que nous deux. Pour la plupart des films nous ne sommes que les deux.

\section{Pourriez-vous nous dire quelques mots sur le financement du film?}

C'est Arte et son éditrice qui ont rendu possible la réalisation de ce film. La télévision suisse aussi, puis BAC Productions. Nous avons eu la possibilité de faire un gonflage en $35 \mathrm{~mm}$. C'était le rêve pour nous, d'avoir une copie $35 \mathrm{~mm}$ de ce film, parce que ça change encore quelque chose. Mais ce n'était pas prévu au début.

\section{Vous l'avez donc tourné en vidéo?}

W. P.: Oui, en mini-DV.

N. H.: Au début, nous souhaitions le tourner en pellicule. La vidéo s'est pourtant révélée plus adéquate que prévu. Dans cette petite chambre, une grande caméra aurait été impratiquable. Le film vit de l'intimité.

W. P.: Finalement, les cadrages ne seraient pas différents si nous avions tourné le film en pellicule.

N. H.: Mais cela tient peut-être à ce que nous sommes avant tout des réalisateurs habitués au support film. Notre usage de la vidéo est différent par rapport à 
quelqu'un qui ne connaît que le support vidéo. On peut sentir cette sorte de tradition du film en pellicule dans nos travaux...

W. P.: Voilà ce que je dis parfois aux étudiants de cinéma: je crois qu'il serait bien, même si ce n'est maintenant plus possible, que chacun de vous doive travailler très dur et en étant mal payé pour acheter une pellicule de film. Une seule - que vous fassiez deux ou trois plans, et que vous deviez travailler de nouveau pour payer le laboratoire et qu'enfin, vous puissiez regarder le résultat. Tu comprends ainsi que chaque plan est quelque chose de précieux. Dès le moment où tu sais cela, tu as un certain respect pour ce que tu filmes. En revanche, avec la vidéo, on peut toujours, si ce n'est pas bien, recommencer le travail. Au-delà de ça, je crois que ça ne concerne pas seulement la qualité d'un plan, c'est aussi une question de respect pour un moment de la vie. Sans caméra, chaque moment de la vie est une chose absolument précieuse. Et ce n'est pas un slogan, c'est une réalité. $\mathrm{Si}$ nous perdons ce respect, nous n'avons de respect ni pour les autres, ni envers nous-mêmes, ni envers la vie. Je constate que la société a perdu le respect pour ce précieux cadeau qui s'appelle la vie.

N. H.: Et aussi l'art de ne pas tourner. Parce que le cinéma implique toujours la décision de tourner ou de ne pas tourner. Avec la vidéo, tu as toujours la tentation de tourner. Renoncer à filmer parce que c'est un moment de pure communication directe, notre usage de la caméra découle de cela.

En debors de votre activité de cinéastes, vous enseignez le cinéma?

De temps en temps. On est invités sur quelques jours pour des workshops. Nous avons enseigné à Bordeaux, à Genève, la dernière fois à Lucerne.

\section{Dans le cadre de Ciné Nomad, produisez-vous d'autres films?}

Non, juste nos films.

W. P.: C’est déjà dur, ça suffit! Nous ne désirons pas produire des travaux d'autres personnes. On doit déjà le faire pour nous. Le faire pour d'autres gens, je ne pourrais pas. Même si parfois des gens viennent avec des projets très intéressants.

Une question plus générale par rapport à la question de documentaire: on qualifie vos films de documentaires, mais vous, comment définiriez-vous vos films? Quel est le terme que vous utiliseriez?

N. H.: Un terme chaque fois différent. Step across the Border: A Ninety Minute Improvisation; Middle of the Moment: Ciné-poème; Brother Yusef: a Chamber film. Il s'agit vraiment de trouver une expression pour la forme spécifique que nous avons essayé de créer. W. P.: Pour échapper aux définitions possibles. N. H.: On essaie toujours de trouver le mot juste.

Quels liens avez-vous avec le milieu cinématographique suisse?

N. H.: Je suis suisse, et la plupart de nos amis proches vivent en Suisse. Les moyens financiers viennent de plus en plus souvent de Suisse, parce qu'en Allemagne il est plus difficile de produire des films comme les nôtres. On a trouvé un certain réseau ici. C'était impossible de trouver des moyens en Allemagne pour Brother Yusef, par exemple.

W. P.: Comme background je dirais que nous ne sommes ni suisses ni allemands. Nous sommes des cinénomades!

N. H.: Au fil de toutes ces années, on a trouvé en Suisse des compagnons comme Peter Mettler, Peter Liechti, ou comme Franz Reichle. Ce sont des amis.

W. P.: C'est une amitié de nombreuses années avec ces gens et on est toujours en échange avec eux.

N. H.: On s'invite pour regarder les différentes versions de montage, par exemple. On a un échange très vivant.

\section{Et avec des cinéastes suisses romands?}

Avec Pascal Magnin, par exemple, Michel Favre, ou encore Fosco et Donatello Dubini. 
W. P.: Avec tous ces amis, nous sommes absolument ouverts. Tu ne peux pas avoir d'amitié sans confiance. Une amitié ne peux pas se développer sans cela. Ça n'est pas une communication intéressante. C'est seulement au moment où tu es vraiment ouvert, où chacun donne tout ce qu'il sait, y compris son opinion sans restriction, que ça devient une communication intéressante. Autrement, pourquoi se parler? Mais je pense aussi que ce monde met de plus en plus les gens en concurrence, spécialement dans le domaine artistique, et encore plus dans le cinéma qui est tellement lié à la finance. Et si on ne trouve pas un moyen de s'ouvrir, de laisser ses peurs en arrière et de réaliser qu'on est tous fondamentalement dans la même situation, je ne pense pas que l'art puisse grandir ou puisse produire des choses intéressantes. Si on est en concurrence, on est dans une logique publicitaire: qui fait la meilleure publicité parvient à placer son produit.

\section{Le fait de faire du documentaire, cela change-t-il quelque chose par rapport à la fiction?}

N. H.: Oui. La solidarité entre documentaristes est beaucoup plus grande qu'entre réalisateurs de fiction.
W. P.: Cela vient aussi du fait que quand vous faites du documentaire, vous êtes nécessairement en étroite relation avec ce qu'on appelle la réalité. La réalité est votre matériel central, même si, d'une certaine manière, vous fictionnalisez la réalité. Car en la regardant, vous en faites déjà de la fiction: pourquoi est-ce que je regarde ceci, et non cela? Il s'agit d'une décision, et donc déjà d'une fiction. Mais je sais combien est fragile le regard, combien est fragile la réalité, et combien j'ai de la chance, vraiment de la chance, de pouvoir attraper quelque chose qui signifie plus que le fait d'être simplement une image. Pour un film de fiction, la réalité est l'ennemi, parce qu'elle est toujours en train d'amener des choses qui ne sont pas censées y être. La réalité doit donc être repoussée de côté par le cinéaste qui veut créer. C’est une attitude différente.

Le dernier chapitre de Brother Yusef s'appelle "Tree», et le dernier plan du film est une porte-fenêtre qui se referme. Cela donne Three Window...

N. H.: Ah, oui, c'est beau! Il y a des liens intérieurs entre les films, c'est beau. On est toujours surpris par les observations des autres! 